\title{
Quantifying functional diversity with graph-theoretical measures: advantages and pitfalls
}

\author{
C. Ricotta ${ }^{1}$ and M. Moretti ${ }^{2}$
}

\author{
${ }^{1}$ Department of Plant Biology, University of Rome "La Sapienza", Piazzale Aldo Moro 5, 00185 Rome, Italy. \\ E-mail: carlo.ricotta@uniroma1.it,Fax:+3906 4457540 \\ ${ }^{2}$ Swiss Federal Research Institute WSL, Research Unit Ecosystem Boundaries, Via Belsoggiorno 22, \\ 6500 Bellinzona, Switzerland. E-mail: marco.moretti@wsl.ch, Fax: +41 0918215239
}

Keywords: Clustering, Minimum spanning tree, Pair-wise species distances.

\begin{abstract}
Recently, a number of measures of functional diversity have been proposed for data on species presences and absences. One of the most fashionable methods uses cluster analysis of species computed from a matrix of functional characters. Functional diversity is then summarized as the sum of branch lengths of the dendrogram (FDD). Like other graph-theoretical measures of functional diversity, FDD is an increasing function of species richness. This makes FDD inadequate for comparative studies if we want to quantify a component of functional diversity that is not directly related to differences in species counts. The aim of this paper is thus to develop a graph-theoretical measure of functional diversity that does not depend of species richness. The edges of the minimum spanning tree, calculated from the pair-wise inter-species dissimilarity matrix based on functional traits, are ranked and then a power law relationship is established with the cumulative distances. We empirically demonstrate that the exponent of this relationship is independent of species richness and is therefore a suitable measure of functional diversity.
\end{abstract}

Abbreviations: FDD-Functional Diversity based on Dendrograms; FDM-Functional Diversity based on Minimum spanning trees; MST-Minimum Spanning Tree.

\section{Introduction}

Juhász-Nagy (1993) first conceived the measurement of community diversity as an iterative process involving successive levels of increasing abstraction (see also Podani et al. 2005, Ricotta 2007). In this view, traditional diversity measures, like the Shannon or the Simpson index, have been recently complemented by measures of functional diversity. Whereas traditional diversity indices focus on species richness and evenness, functional diversity has been defined by Tilman (2001) as "those components of biodiversity that influence how an ecosystem operates or functions".

Several types of functional diversity measures have been proposed, like the sum of pair-wise species functional distances (Walker et al. 1999), Rao's quadratic diversity (BottaDukát 2005, Ricotta 2005), or the measures developed by Solow and Polasky (1994), Mason et al. (2005), Heemsbergen et al. (2004), Ricotta (2004), Mouillot et al. (2005), de Bello et al. $(2006,2007)$ and Lepš et al. (2006). For species presence and absence scores, Petchey and Gaston (2002, 2006) applied cluster analysis of species calculated from a matrix of functional characters, and then used the sum of branch lengths of the dendrogram as a multivariate measure of functional diversity (FDD).
From an operational viewpoint, Podani and Schmera (2006) suggested Gower's formula and group average (UPGMA) clustering as a standard combination of techniques for calculating FDD. From a more theoretical viewpoint, the graph-theoretical definition of functional diversity proposed by Petchey and Gaston (2002) is however, a more complex problem than previously thought and several questions remain open (see Petchey and Gaston 2006, Podani and Schmera 2006). For instance, the idea of developing a dendrogram-based measure of functional diversity was inspired by evolutionary biology (e.g., Faith 1992), where the total length of a phylogenetic tree is used as a measure of phylogenetic diversity of a given set of species (see Petchey and Gaston 2002, 2006). Whereas phylogenies generally possess a non-overlapping hierarchical structure, functional diversity tends to have a non-hierarchical, overlapping one (Von Euler and Svensson 2001, da Silva and Batalha 2006). Evolutionary relationships among taxa can be represented in terms of tree graphs, such that the terminal nodes of a phylogenetic tree embody the taxa studied, while the interior nodes of the tree represent 'hypothetical taxonomic units' (i.e., the hypothetically most recent common ancestor of all descendent taxa in the phylogenetic tree; Farris 1970). Finally, the edges (i.e., branches) of the phylogenetic tree indicate the evolutionary pathways (i.e., the ancestor $\rightarrow$ descendant relationships) of all tree nodes. By contrast, from a functional per- 
spective, the hierarchical structure of dendrogram-based diversity measures is much less interpretable than its phylogenetic counterpart. As noted by Petchey and Gaston (2006), functional diversity measures based on hierarchies will be less appropriate when real functional relationships are nonhierarchical.

Also, it is easily shown that FDD is an increasing function of species richness. This effect renders FDD inadequate if we want to compare functional diversity of different communities independently of differences in species richness. The aim of this paper is thus to develop a graph-theoretical measure of functional diversity that is independent of species richness. Such a measure makes sense specifically for comparative studies for which a suitable quantification of that component of functional diversity is needed, which is not directly related to differences in species counts.

\section{Can we develop a graph-theoretical measure of functional diversity that is independent of species richness?}

Weitzman (1992) proposed measuring (graph-theoretical) diversity with a dynamic programming recursion that can be represented by an unrooted spanning tree, the nodes of which correspond to the $S$ species in the assemblage. Unfortunately, due to its computational complexity, the Weitzman diversity function is principally of theoretical interest, remaining mostly outside the mainstream of studies on community diversity.

Nonetheless, besides the Weitzman tree, other types of tree graphs, like the 'minimum spanning tree' (MST) may prove useful in revealing the functional structure of a given species assemblage. Like dendrograms obtained from hierarchical clustering, the MST is derived from the matrix of pairwise species functional distances $d_{i j}$. However, in MSTs, each vertex corresponds to an object (species), so there are no 'abstract' vertices in the graph. For $S$ species, MSTs are composed of $S$-1 edges, each weighted by the corresponding distance value, such that the total sum of edge length is minimized (Podani 2000). For an overview of the relationships between minimum spanning trees and single linkage hierarchical classifications, see Gower and Ross (1969).

Based on MSTs, we can thus calculate another graphtheoretical measure of functional diversity, FDM, that is defined simply as the sum of branch lengths of the corresponding MST. Figure 1 shows how both graph-theoretical measures of functional diversity, FDD and FDM are computed from the matrix of pair-wise species functional distances of Table 1, together with the corresponding trees.

Unfortunately, like for FDD, the values of FDM increase with species richness as a necessary mathematical outcome of the definition of functional diversity. For instance, the number of edges in a MST is a trivial linear function of the number of species in the assemblage. Likewise, a perfectly dichotomous (i.e., bifurcating) dendrogram obtained from hierarchical clustering of $S$ species possesses $2 S-2$ edges.
Also, from a probabilistic point of view, plant mixtures of higher-richness assembled at random from a pool of species have a greater chance of containing a combination of species with complementary traits. This effect is similar to the "selection effect" discussed in studies of functional biodiversity effects (e.g., Loreau and Hector 2001; see also Lepš et al. 2006).

The mutual dependence of species richness and functional diversity is obviously influenced by the inherent multidimensional data structure. For instance, some species are very similar to each other, while some are very different. Therefore, though species richness has been usually considered a proxy for functional diversity, this relationship is not expected to be very strong (Díaz and Cabido 2001, Petchey and Gaston 2006). On the other hand, if the relationship between functional diversity and species richness is marked, then differences in functional diversity values among different species assemblages could simply reflect differences in the species count rather than any fundamental difference in the functional organization of the assemblages. As a consequence, graph-theoretical measures of functional diversity coming from different assemblages can be compared only if some normalization is used to account for the underlying species number effect (Podani and Schmera 2006).

Intuitively, a straightforward solution for normalizing FDD and FDM would be to divide these functional measures
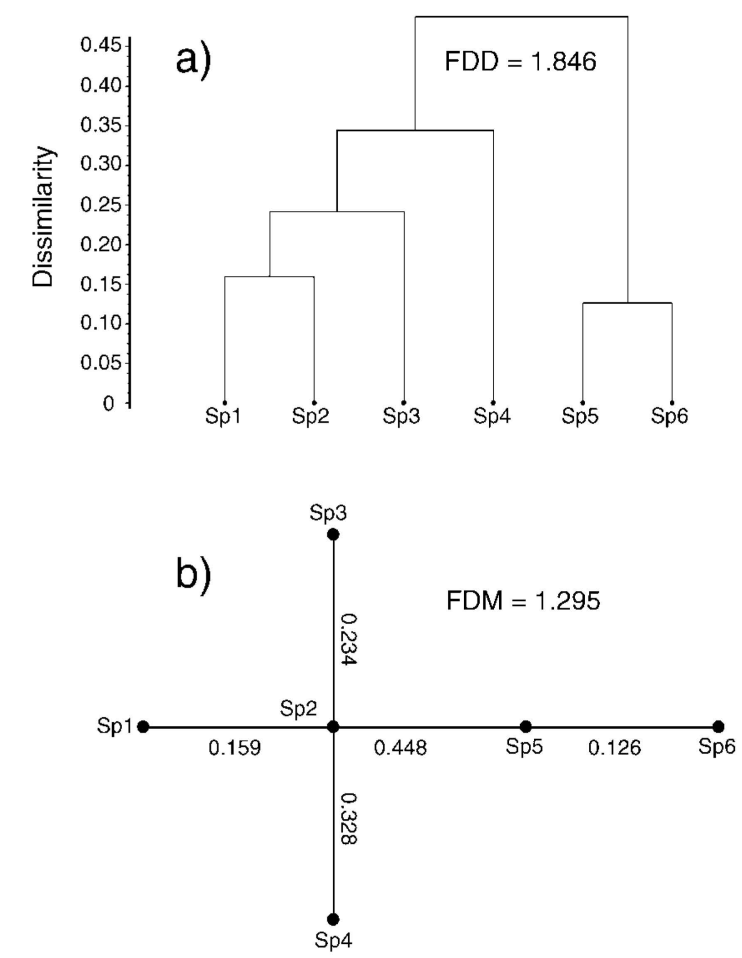

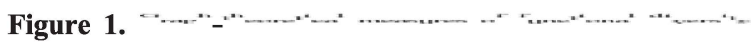

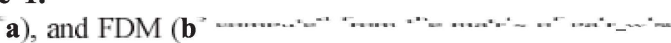
-

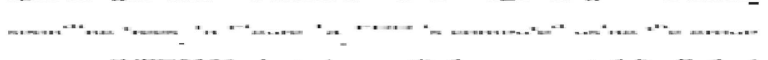

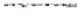

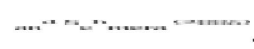


Table 1

\begin{tabular}{lllllll}
\multicolumn{1}{c}{} & & & & & \\
\hline & Sp 1 & Sp 2 & Sp 3 & Sp 4 & Sp 5 & Sp 6 \\
\hline Species 1 & 0 & 0.159 & 0.250 & 0.349 & 0.495 & 0.513 \\
Species 2 & 0.159 & 0 & 0.234 & 0.328 & 0.448 & 0.448 \\
Species 3 & 0.250 & 0.234 & 0 & 0.357 & 0.532 & 0.498 \\
Species 4 & 0.349 & 0.328 & 0.357 & 0 & 0.477 & 0.488 \\
Species 5 & 0.495 & 0.448 & 0.532 & 0.477 & 0 & 0.126 \\
Species 6 & 0.513 & 0.448 & 0.498 & 0.488 & 0.126 & 0 \\
\hline
\end{tabular}

by the number of edges in the corresponding trees (i.e., $S$-1 for FDM and $2 S-2$ for FDD). Here, it is worth noting that polytomous (i.e., multifurcating) dendrograms with less than $2 S-2$ edges can be interpreted as a special class of dichotomous trees in which some edges have zero length.

However, a simple division of functional diversity by the number of tree edges is not adequate because the relationship between functional diversity and the number of edges is not linear but rather steep initially and then it later flattens out. This is because species-rich assemblages tend to be more functionally redundant, on average, than species-poor communities (see Fonseca and Ganade 2001). That is, by adding new species to the assemblage, the functional redundancy becomes increasingly higher, so that a non-linear relationship between functional diversity and species richness is obtained. Hence, dividing FDM by $S-1$ or $F D D$ by $2 S-2$ overcompensates the influence of species richness at high levels of $S$.

As an alternative, a Monte Carlo rarefaction approach can be used in which more species-rich assemblages are subsampled at random to artificially obtain assemblages of similar richness (see Podani and Schmera 2006). This procedure can be repeated many times to calculate confidence intervals and hence to evaluate the statistical differences between the observed functional diversity values. However, this approach also has its limitations if the number of species between the compared assemblages is very highly divergent because this would mean "losing" the majority of functional information in the more species-rich assemblages.

A different approach consists in establishing a non-linear relationship between functional diversity and species richness, similar to the equations used to describe species-area relationships (see, e.g., Tjørve 2003). Due to the presence of interior nodes without any clear ecological meaning in dendrograms obtained from hierarchical clustering, we feel that we are far from finding a simple, meaningful solution to this problem for FDD. By contrast, for FDM the answer seems to be easier.

Though there is no theory to predict the exact shape of this relationship, we can start with the observation that the construction of the MST can be envisioned as a sequential addition of pair-wise species functional distances $d_{j}$ of increasingly larger size, continuing until $S-1$ distances (i.e., edges) are included in the spanning tree. We empirically found out that the best model for parametrizing the scaling behavior of functional diversity $v s$. species richness in the minimum spanning tree is a power-law between edge rank $r\left(d_{i j}\right)$ and the corresponding cumulative edge length:

$\operatorname{FDM}(\leq \mathrm{r}) \propto r\left(d_{i j}\right)^{\alpha}$

where the shortest pair-wise species distance $d_{j}$ in the spanning tree is assigned the rank $r=1$, the second shortest distance has rank $r=2$, and so on until $S-1$ distances are sequentially added to the spanning tree, and $\operatorname{FDM}(\leq r)$ is the cumulative functional diversity obtained by adding all pairwise species distances $d_{i j}$ with rank $\leq r$. See Figure 2, for an example.

The exponent $\alpha$ in Eq. 1 is independent of the richness of the assemblage and summarizes how fast the functional diversity increases when edges are sequentially added to the spanning tree. In a sense, the values of $\alpha$ are a richness-independent measure of functional redundancy (i.e., the amount of species functional overlap in trait space); low values of $\alpha$ denote high functional redundancy, while high values of $\alpha$ denote high functional diversity.

\section{Illustrative example}

To investigate the influence of species richness on functional diversity we used plant assemblages. Plants were recorded in 12 study sites along a uniform south-facing slope

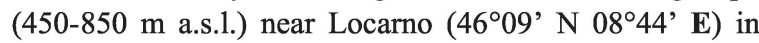
Southern Switzerland. The vegetation is dominated by former coppice stands of European chestnut (Castanea sativa Mill.) on acid soil. For more details, see Moretti et al. (2006). For each study site, the presence/absence scores of all vascular plants within three $10 \mathrm{~m} \times 10 \mathrm{~m}$ quadrats were recorded. The quadrats were sampled in May-June and again in August in order to identify as many species as possible and to include species with different phenology. Species richness in the sampled assemblages varied from 15 to 41 .

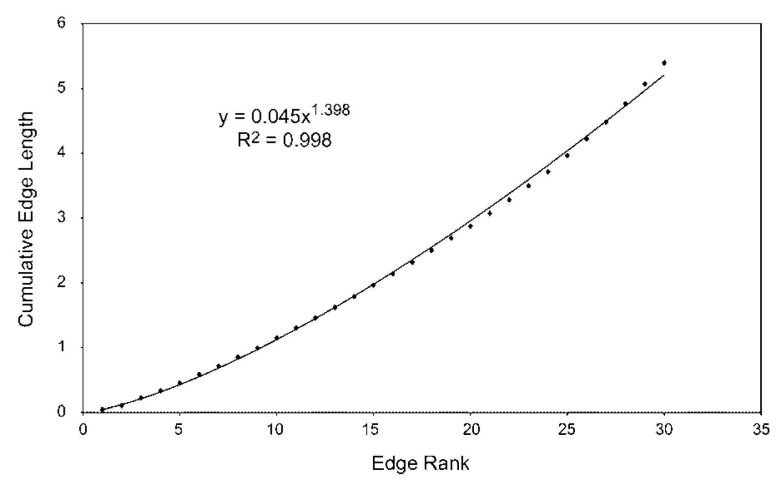

Figure 2

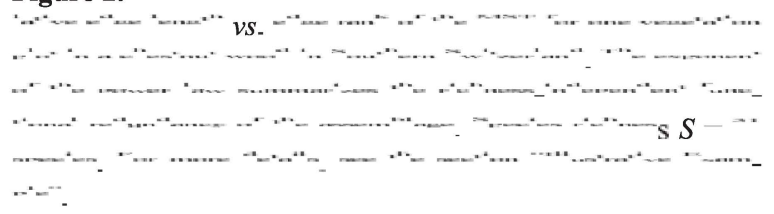


Table 2.

\begin{tabular}{|c|c|c|}
\hline Functional Trait & Data type & Attribute \\
\hline Habitat Type & Nominal & $\begin{array}{l}\text { Most commonly present in: rocky areas; cultivated areas; meadows; } \\
\text { shrublands; forests }\end{array}$ \\
\hline $\begin{array}{l}\text { Agent of } \\
\text { dispersal }\end{array}$ & Nominal & Zoochorous; Anemochorous \\
\hline Pollen vector* & Nominal & Pollinated by wind; Pollinated by animals \\
\hline CSR strategy & Nominal & Competitor; Stress tolerant; Ruderal \\
\hline Maximum height & Ordinal & $\begin{array}{l}\text { 1. Herbs and sub-shrubs }(\leq 150 \mathrm{~cm}) ; 2 \text {. Shrubs }(>150-300 \mathrm{~cm}) \text {; } \\
\text { 3. Trees }(>300 \mathrm{~cm})\end{array}$ \\
\hline Seed weight & Ordinal & $\begin{array}{l}\text { 0. No seed produced; } 1 \text {. Seed too small to be measured; } 2 \leq 0.2 \mathrm{mg} \text {; } \\
\text { 3. } 0.2-0.5 \mathrm{mg} ; 4,0.5-1.0 \mathrm{mg} ; 5,1.0-2.0 \mathrm{mg} ; 6,2.0-10,0 \mathrm{mg} ; 7,>10 \mathrm{mg}\end{array}$ \\
\hline Leaf persistence & Ordinal & 1. Aestival green; 2. Partial evergreen; 3 . Evergreen \\
\hline Life history & Ordinal & 1. Annual; 2 . Usually biennial; 3 . Perennial \\
\hline Life form & Ordinal & $\begin{array}{l}\text { 1. Phanerophyte and Nano-Phanerophyte; } 2 \text { Phanerophyte- } \\
\text { Chamaephyte; } 3 \text {. Chamaephyte; } 4 \text {. Chamaephyte-Hemicryptophyte; } \\
\text { 5. Hemicryptophyte; } 6 \text {. Hemicryptophyte-Geophyte; } 7 \text {. Geophyte; } \\
\text { 8. Therophyte }\end{array}$ \\
\hline Lateral spread & Ordinal & $\begin{array}{l}\text { 1. Therophytes, lateral spread of exceedingly limited extent and } \\
\text { duration; } \\
\text { 2. Perennials with compact unbranched rhizomes or forming small } \\
\text { tussocks }(\varnothing<10 \mathrm{~cm}) ; 3 \text {. Perennials with rhizomatous systems or large } \\
\text { tussocks }(\varnothing 10-25 \mathrm{~cm}) ; 4 \text {. Perennials attaining } \varnothing 25-100 \mathrm{~cm} \text {; } \\
\text { 5. Perennials attaining } \varnothing>100 \mathrm{~cm}\end{array}$ \\
\hline $\begin{array}{l}\text { Start of the } \\
\text { flowering } \\
\text { season }\end{array}$ & Quantitative & $\mathrm{cos}$ \\
\hline $\begin{array}{l}\text { Duration of the } \\
\text { flowering } \\
\text { season }\end{array}$ & Quantitative & Number of months \\
\hline
\end{tabular}

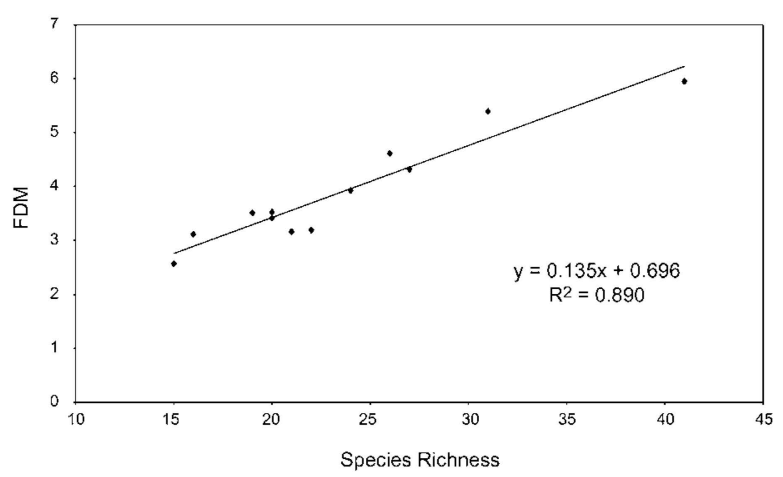

Figure 3.

Based on twelve widely used functional traits relevant to most taxa (see Table 2), we calculated a pair-wise species distance matrix for each study site. Pair-wise species distances were computed using Gower's formula (Gower 1971), modified by Podani (1999) to allow the inclusion of ordinal variables in the data.

Next, from the pair-wise species distance matrices of each site, we calculated all graph-theoretical measures of functional diversity discussed above, FDD, FDM, and $\alpha$ (i.e., the exponent of the power law of Eq. 1). FDM and $\alpha$ were both computed from the output data of the SYN-TAX 2000 program package (Podani 2001); FDD was computed with a small routine written by Podani (unpublished; courtesy of the author) for calculating tree lengths based on UPGMA clustering performed by SYN-TAX.

The plot of FDM vs species richness of the analyzed assemblages (Figure 3 ) shows a very strong positive correla-

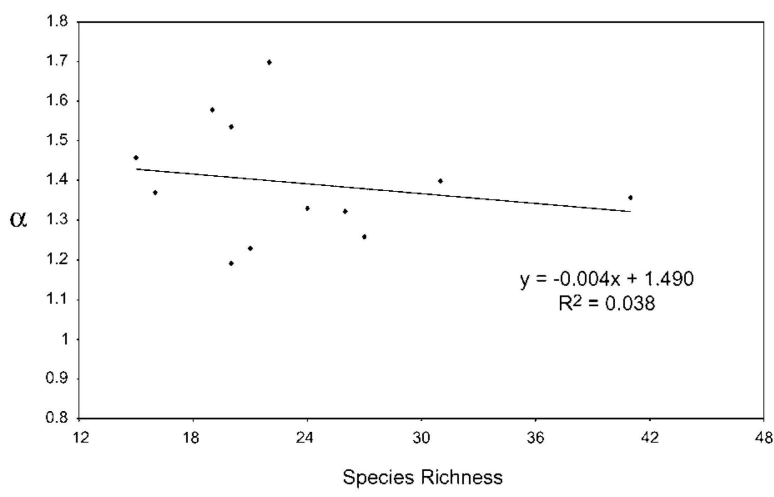

Figure 4 $\alpha v s \cdots \cdots$ tion that makes FDM inadequate for comparing the functional diversity of assemblages with a different number of species. The qualitative trend obtained from the plot of FDD vs. species richness is very similar to the trend presented in Figure 3, and is therefore not shown here. By contrast, as shown in Figure 4, the exponent $\alpha$ summarizes the functional redundancy/diversity of the plant assemblages without being dependent on species richness $\left(R^{2}=0.038, p=0.541\right)$.

\section{Discussion}

As shown by a number of studies (e.g., Ricotta 2005, Petchey and Gaston 2006, Podani and Schmera 2006), the measurement of functional diversity is a complex question that requires a complex answer. Therefore, an accurate evaluation of diversity measures in terms of their basic properties is a necessary condition for answering the question: "Does the index measure what we want to measure?" (see 
Sherwin et al. 2006). In this view, a clear understanding of the mathematics behind the graph-theoretical measures of functional diversity is the only meaningful way to assess whether these measures allow useful biological distinctions between different species assemblages (see Hill 1997, Mouillot and Wilson 2002).

The proposed index seems a promising tool for comparing the species-independent functional diversity of assemblages of different richness, for example, in biodiversity experiments. In classical biodiversity experiments, species assemblages of different richness are constructed and the observed responses of ecosystem processes (usually the aboveground biomass as a proxy of productivity) are measured. The interpretation of these experiments, however, has been widely disputed because two types of effects may operate simultaneously. For instance, as the number of species increases in ecological communities so does the range of 'functional space' occupied by the community (Lawton et al. 1998). In this context, the use of species-independent measures of functional diversity may be capable of disentangling the effects of species richness and functional redundancy when investigating the mechanisms behind diversity effects on ecosystem processes.

From a mathematical viewpoint, instead of using a power-law for parametrizing the relationship between functional diversity and species richness in the minimum spanning tree, several alternative scaling models that exhibit a curvature in log-log space may be used (e.g., Mandelbrot 1983, Laherrère 1996, Landini and Rigaut 1997, Laherrère and Sornette 1998). Most of these models, such as the ZipfMandelbrot function (Mandelbrot 1983), or the parabolic fractal (Laherrère 1996) are generalizations of Eq. 1 that recover a pure power law distribution for an appropriate tuning of the adjustable parameters. Therefore, additional work is needed to test the performance of these models in describing the non-linear scaling behavior of functional diversity as a function of species richness. Overall, we hope this paper will provide valuable insights into the potentials and limits of graph-theoretical measures of functional diversity.

\section{References}

Botta-Dukát, Z. 2005. Rao's quadratic entropy as a measure of functional diversity based on multiple traits. J. Veg. Sci. 16: 533-540.

da Silva, I.A. and M.A. Batalha. 2006. Taxonomic distinctness and diversity of a hyperseasonal savanna in Central Brazil. Divers. Distrib. 12: 725-730.

Díaz, S. and M. Cabido. 2001. Vive la difference: plant functional diversity matters to ecosystem processes. Trends Ecol. Evol. 16: 646-650.

de Bello, F., J. Lepš, S. Lavorel and M. Moretti. 2007. Importance of species abundance for assessment of trait composition: an example based on pollinator communities. Community Ecol. 8: 163170.

de Bello, F., J. Lepš and M.T. Sebastià. 2006. Variations in species and functional plant diversity along climatic and grazing gradients. Ecography 29: 801-810.
Faith, D.P. 1992. Conservation evaluation and phylogenic diversity. Biol. Cons. 61: 1-10.

Farris, J.S. 1970. Methods for computing Wagner trees. Syst. Zool. 19: 83-92.

Fonseca, C.R. and G. Ganade. 2001. Species functional redundancy, random exinctions and the stability of ecosystems. J. Ecol. 89: 118-125.

Gower, J.C. 1971. A general coefficient of similarity and some of its properties. Biometrics 27: 857-874.

Gower, J.C. and G.J.S. Ross. 1969. Minimum spanning trees and single linkage cluster analysis. Appl. Stat. 18: 54-64.

Heemsbergen, D.A., M.P. Berg, M. Loreau, J.R. van Hal, J.H. Faber and H.A. Verhoef. 2004. Biodiversity effects on soil processes explained by intraspecific functional dissimilarity. Science 306 : 1019.

Hill, M.O. 1997. An evenness statistic based on the abundanceweighted variance of species proportions. Oikos 79: 413-416.

Juhász-Nagy, P. 1993. Notes on compositional diversity. Hydrobiologia 249: 173-182.

Laherrère, J.H. 1996. Distributions de type fractal parabolique dans la Nature. C. R. Acad. Sci. Paris II 322: 535-541.

Laherrère, J.H. and D. Sornette. 1998. Stretched exponential distributions in nature and economy: "Fat tails" with characteristic scales. Eur. Phys. J. B 2: 525-539.

Landini, G. and J.P. Rigaut. 1997. A method for estimating the dimension of asymptotic fractal sets. Bioimaging 5: 65-70.

Lawton, J.H., S. Naeem, L.J. Thompson, A. Hector and M.J. Crawley. 1998. Biodiversity and ecosystem function: getting the Ecotron experiment in its correct context. Funct. Ecol. 12: 848852.

Lepš, J., F. de Bello, S. Lavorel and S. Berman. 2006. Quantifying and interpreting functional diversity of natural communities: practical considerations matter. Preslia 78: 481-501.

Loreau, M. and A. Hector. 2001. Partitioning selection and complementarity in biodiversity experiments. Nature 412: 72-76.

Mandelbrot, B.B. 1983. The Fractal Geometry of Nature. Freeman, San Francisco.

Mason, N.V.H., D. Mouillot, W.G. Lee and J.B. Wilson. 2005. Functional richness, functional evenness and functional divergence: the primary components of functional diversity. Oikos 111: 112118.

Moretti, M., P. Duelli, K.M. Obrist. 2006. Biodiversity and resilience of arthropod communities after fire disturbance in temperate forests. Oecologia 149: 312-327.

Mouillot, D., N.W.H. Mason, O. Dumay and J.B. Wilson. 2005. Functional regularity: a neglected aspect of functional diversity. Oecologia 142: 353-359.

Mouillot, D. and J.B. Wilson. 2002. Can we tell how a community was constructed? A comparison of five evenness indices for their ability to identify theoretical models of community construction. Theor. Popul. Biol. 61: 141-151.

Petchey, O.L. and K.J. Gaston. 2002. Functional diversity (FD), species richness and community composition. Ecol. Lett. 5: 402411.

Petchey, O.L. and K.J. Gaston. 2006. Functional diversity: back to basics and looking forward. Ecol. Lett. 9: 741-758.

Podani., J. 1999. Extending Gower's general coefficient of similarity to ordinal characters. Taxon 48: 331-340.

Podani. J. 2000. Introduction to the Exploration of Multivariate Biological Data. Backhuys Publishers, Leiden. 
Podani, J. 2001. SYN-TAX 2000. Computer Programs for Data Analysis in Ecology and Systematics. User's Manual. Scientia, Budapest.

Podani, J., P. Csontos, J. Tamás and I. Miklós. 2005. A new multivariate approach to studying temporal changes of vegetation. Plant. Ecol. 181: 1-16.

Podani, J. and D. Schmera. 2006. On dendrogram-based measures of functional diversity. Oikos 115: 179-185.

Ricotta, C. 2004. A parametric diversity measure combining the relative abundances and taxonomic distinctiveness of species. $\mathrm{Di}$ vers. Distrib. 10: 143-146.

Ricotta, C. 2005. A note on functional diversity measures. Basic Appl. Ecol. 6: 479-486.

Ricotta, C. 2007. A semantic taxonomy for diversity measures. Acta Biotheor. 55: 23-33.

Sherwin, W.B., F. Jabot, R. Rush and M. Rossetto. 2006. Measurement of biological information with applications from genes to landscapes. Mol. Ecol. 15: 2857-2869.
Solow, A.R. and S. Polasky. 1994. Measuring biological diversity. Environ. Ecol. Stat. 1: 95-107.

Tilman, D. 2001. Functional diversity. In: S.A.Levin (ed.), Encyclopedia of Biodiversity. Academic Press, San Diego, pp. 109-120.

Tjørve, E. 2003. Shapes and functions of species-area curves: a review of possible models. J. Biogeogr. 30: 827-835.

Von Euler, F. and S. Svensson. 2001. Taxonomic distinctness and species richness as measures of functional structure in bird assemblages. Oecologia 129: 304-311.

Walker, B., A.P. Kinzig and J. Langridge. 1999. Plant attribute diversity, resilience, and ecosystem function: the nature and significance of dominant and minor species. Ecosystems 2: 95-113.

Weitzman, M.L. 1992. On diversity. Quart. J. Econ. 107: 363-405.

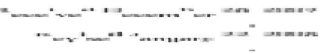

